\title{
RADIONUCLÍDEOS COMO MARCADORES DE UM NOVO TEMPO: O ANTROPOCENO
}

\author{
Cleyton Martins da Silva ${ }^{\circledR a, b}$, Graciela Arbilla ${ }^{a, *,(1)}$, Wilson Machado M Ricardo Soares $^{c, d}$ \\ a'Departamento de Físico-Química, Instituto de Química, Universidade Federal do Rio de Janeiro, 21941-909 Rio de Janeiro - RJ, \\ Brasil

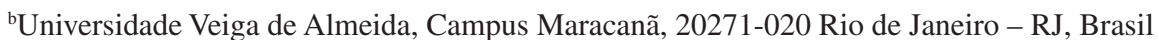 \\ 'Departamento de Geoquímica, Instituto de Química, Universidade Federal Fluminense, Outeiro São João Batista, s/n, 24020-141 \\ Niterói - RJ, Brasil \\ ${ }^{\mathrm{d} I n s t i t u t o}$ Estadual do Ambiente, Avenida Venezuela 110, 20081-312 Rio de Janeiro - RJ, Brasil
}

Recebido em 16/10/2019; aceito em 13/01/2020; publicado na web em 31/03/2020

\begin{abstract}
RADIONUCLIDES AS MARKERS OF A NEW TIME: THE ANTHROPOCENE. On May 2019, the members of the Anthropocene Working Group completed a binding vote to affirm some of the key questions that have been agreed in 2016: the Anthropocene should be considered as a formal chronostratigraphic unit, defined by a Global Boundary Stratotype Section and Point (GSSP), using one of the stratigraphic signals around the mid-twentieth century of the Common Era. The fallout from nuclear weapons testing appears as the most suitable GSSP. In this article, the main anthropogenic markers are discussed as well as the sources or human-made radiation and the signature of nuclear weapons testing. The appearance of ${ }^{239+240} \mathrm{Pu}$ in the early 1950 s and the clear peak, in 1963 , makes a good marker. High resolution archives include layers of sedimentary rocks, lacustrine and marine sediments and corals and polar ice cores. A coupled application of other radioactive isotopes, as ${ }^{137} \mathrm{Cs},{ }^{14} \mathrm{C}$ and ${ }^{241} \mathrm{Am}$, may be useful to categorize distinct fallout signatures. A site to define the Anthropocene (golden spike) would ideally be located between $30^{\circ}$ and $60^{\circ}$ north of the equator. Nevertheless, results obtained in sediments on the Brazilian coast showed that both ${ }^{239+240} \mathrm{Pu}$ and ${ }^{137} \mathrm{Cs}$ signatures could be detected and quantified as markers of this "atomic age".
\end{abstract}

Keywords: Anthropocene; The Great Acceleration; nuclear fallout; ${ }^{137} \mathrm{Cs}$; Chernobyl.

\section{INTRODUÇÃO}

Importa realmente saber em que Época geológica a humanidade está vivendo? Certamente esse é um assunto fundamental para a Geologia e outras áreas afins nas quais a escala de tempo geológico e a Tabela Cronoestratigráfica Internacional são as bases para os estudos dos eventos que marcam as mudanças do Planeta. ${ }^{1-3} \mathrm{Em}$ outras áreas da ciência essa discussão abrange, também, outras conotações inerentes às ciências naturais, políticas, econômicas e sociais. ${ }^{4-6}$ Um número cada vez maior de cientistas concorda que existem evidências claras de mudanças no Sistema Terra, além da variabilidade típica do Holoceno, ocasionadas pelas atividades humanas. O conceito do Antropoceno como um novo tempo no qual o homem tenha se transformado em uma força geológica capaz de mudar o destino, não só da própria espécie, mas também de todas as espécies, foi introduzido por Paul Crutzen e Eugene Stoermer no ano $2000 .^{7,8}$

Inicialmente, o Antropoceno foi tratado pela comunidade que estuda as ciências naturais de uma maneira informal ao considerar diversos assuntos como mudanças climáticas globais, acidificação dos oceanos, biodiversidade, alteração dos ciclos biogeoquímicos do nitrogênio e fósforo, além da produção e acumulação de novos materiais antropogênicos (tecnofósseis). Contudo, em pouco tempo a discussão foi levada à esfera da geologia e, no ano de 2009, foi formado um grupo de trabalho com 38 acadêmicos, representantes de diferentes áreas, como geologia, química, biologia, arqueologia e história, por solicitação da Subcomissão de Estratigrafia do Quaternário (Subcommission of Quaternary Stratigraphy, SQS) da Comissão Internacional de Estratigrafia (International Comission of Stratigraphy, ICS) da União Internacional de Ciências Geológicas

*e-mail: gracielaiq@gmail.com
(International Union Geological Sciences, IUGS), para estudar as possíveis evidências geológicas do Antropoceno que pudessem levar à sua formalização. Os resultados obtidos por esse grupo, chamado de Grupo de Trabalho do Antropoceno (Anthropocene Working Group, $A G W$ ), têm sido divulgados por Newsletters anuais, livros e dezenas de publicações científicas, ${ }^{9}$ e foram apresentados no $35^{\circ}$ Congresso Geológico Internacional, na Cidade do Cabo (África do Sul), ${ }^{10} \mathrm{em}$ 2016, e, posteriormente, compilados em um artigo publicado na revista Anthropocene em 2017 e no livro The Anthropocene as a Geological Unit publicado em 2019. ${ }^{11,12}$

Recentemente, seguindo as recomendações da Subcomissão de Estratigrafia do Quaternário e da Comissão Internacional de Estratigrafia, o Grupo de Trabalho do Antropoceno votou, em forma vinculativa, as propostas da reunião de 2016, na Cidade do Cabo (Figura 1). Em 21 de maio de 2019 foi informado o resultado da votação de 33 membros (97\% do total atual de 34 membros), na qual 29 cientistas ( $88 \%$ do total) votaram a favor da proposta que o Antropoceno seja considerado formalmente como uma unidade cronoestratigráfica com início aproximado na metade do século $\mathrm{XX} \cdot{ }^{13} \mathrm{~A}$ partir dessa votação, essa passou a ser considerada a posição oficial do Grupo de Trabalho que irá guiar o trabalho subsequente de analisar as evidencias científicas para estabelecer um marcador geológico adequado. ${ }^{9} \mathrm{Na}$ Figura 1 são ilustradas as etapas necessárias para que o Antropoceno seja reconhecido oficialmente como Época geológica.

O objetivo deste artigo é discutir o Antropoceno desde o ponto de vista da estratigrafia, apresentando as possibilidades que permitiriam definir seu início como Época, seguindo os procedimentos formalmente aceitos para a construção da escala geológica e, em particular discutir a proposta mais aceita atualmente de usar um marcador radiativo oriundo do fallout das detonações nucleares atmosféricas do século XX. 

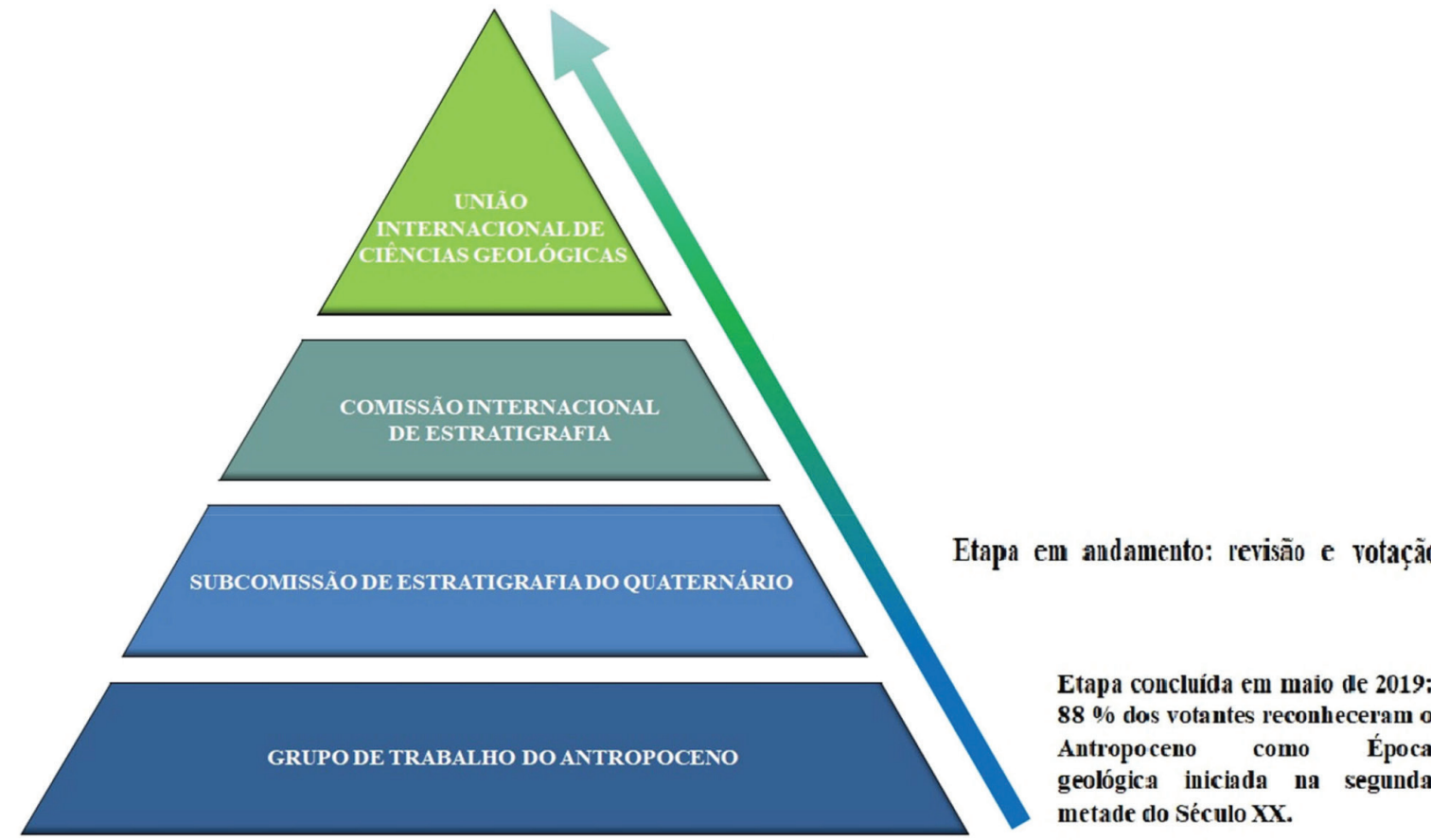

Figura 1. Percurso a ser percorrido pela proposta do Antropoceno para ser reconhecido oficialmente como Época geológica

\section{O INÍCIO DO ANTROPOCENO}

Em um artigo recentemente publicado na Revista Virtual de Química, ${ }^{8}$ Silva e Arbilla apresentaram as diversas propostas sobre o início do Antropoceno, que abrangem desde o início da agricultura até os eventos relacionados ao período histórico informalmente reconhecido como a "Grande Aceleração". Já no livro publicado, também em $2018,{ }^{14}$ o geógrafo Simon Lewis e o geólogo Mark Maslin discutiram o Antropoceno na sua dimensão geológica, ambiental, histórica e cultural através das revoluções energéticas (o início da agricultura e a Revolução Industrial) e os grandes eventos de globalização (a chegada dos conquistadores europeus ao continente Americano e a Grande Aceleração). Entretanto, a determinação do início do Antropoceno no contexto da Geologia está vinculada aos critérios pelos quais são estabelecidas as divisões da Escala de Tempo Geológico.

\section{Estratigrafia e a escala geológica}

Os 4,5 bilhões de anos da história da Terra estão representados na Tabela Cronoestratigráfica Internacional, chamada informalmente de Escala de Tempo Geológico Internacional (International Geologic Time Scale), em que as unidades de tempo (Eon, Era, Períodos, Épocas, Idades) expressam a história geológica da Terra. ${ }^{15}$ Essencialmente, a história da Terra é reconstruída a partir das informações químicas, físicas e biológicas obtidas principalmente nos estratos sedimentares, que preservam os registros dos sucessivos eventos através do tempo. O termo estratigrafia está referido ao estudo da história geológica a partir dos estratos (ou camada) de rochas.

Há duas formas paralelas de classificar a história do Planeta: desde um ponto de vista geocronológico, onde o tempo é dividido em intervalos nos quais aconteceram certos eventos, ou considerando um critério cronoestratigráfico com base nos registros contidos nos estratos que preservam essa história. Ambos os critérios são complementares e paralelos. Ao definir uma unidade de tempo dentro da Tabela Cronoestratigráfica Internacional, um dos aspectos mais importantes é a determinação do seu início a partir de uma posição específica nos registros sedimentares, que define uma base sincrônica, que seja igual no tempo em todos os lugares do planeta, ou eventualmente uma data específica.

Os estratos podem ser divididos com base em suas características físicas em unidades litoestratigráficas, as quais podem ser sincrônicas (aproximadamente da mesma época em diferentes lugares) ou diacrônicas (pertencendo substancialmente a tempos diferentes em localidades diferentes). ${ }^{12}$ A classificação dos estratos pode ser realizada usando diferentes métodos, como a caracterização das relações isotópicas, fósseis, propriedades magnéticas e eventos globais.

Em geral, são utilizados dois tipos de referências no tempo: Global Standard Stratigraphic Ages (GSSAs) e Global Boundary Stratotype Sections and Points (GSSPs), comumente chamados "Golden spikes". ${ }^{12}$ Para os registros estratigráficos mais antigos, nos quais é mais difícil uma determinação precisa, os inícios foram estabelecidos em termos de datas (GSSA). Para estabelecer um GSSP deve ser escolhido um nível dentro da unidade estratigráfica, no qual exista um registro claro, por exemplo, o aparecimento de um sinal químico ou um fóssil, que possa ser caracterizado.

A título de comparação, o início da Época Holoceno foi definido como o fim da era glacial, por GSSA, sendo ainda 10.000 anos BP (onde BP significa "before present", considerado na geologia como o 1 de janeiro de 1950). Em maio de 2008, foi ratificado, por GSSP, como 11.700 b2k (antes de 2000 d.C.), com um erro máximo de 99 anos, a partir de registros de gelo obtidos no Projeto NGRIP na Groenlândia (North Greenland Ice Core Project), a 1.492,25 m de profundidade, que permitiram identificar claramente uma mudança na contribuição isotópica do deutério $\left({ }^{2} \mathrm{H} /{ }^{1} \mathrm{H}\right)$, com uma diminuição de aproximadamente 2 - 3\%o na transição Pleistoceno-Holoceno, que corresponde a uma diminuição na temperatura superficial dos oceanos 
de 2 - $4{ }^{\circ} \mathrm{C}$. Outros registros auxiliares, como a mudança na relação dos isótopos ${ }^{18} \mathrm{O} /{ }^{16} \mathrm{O}$ e as concentrações de $\mathrm{Na}^{+}$, permitiram ratificar a determinação do início do Holoceno, em um trabalho detalhado realizado durante quatro anos pelo grupo de trabalho coordenado pelo Professor Mike Walker (University of Wales). ${ }^{16}$

A determinação do início do Antropoceno parece mais difícil e é objeto de amplo debate na literatura desde o início da sua proposta. ${ }^{11,12,14,17-21}$ Assim, considerando o enfoque geocronológico e cronoestratigráfico, apesar do relativamente pouco tempo transcorrido, é possível analisar o início do Antropoceno a partir dos dados históricos, que envolvem diversos eventos relacionados às ciências naturais, com os registros estratigráficos, enquanto a heterogeneidade da ocorrências de tais eventos dificulta a determinação de marcadores sincrônicos em diversos pontos do Planeta. ${ }^{17}$

Atualmente, a maioria dos cientistas concorda que existem evidências suficientes indicando que o Antropoceno é uma nova Época, diferente do Holoceno, e que existem marcadores estratigráficos suficientes, conforme confirmado pela recente votação do Grupo de Trabalho do Antropoceno. ${ }^{9,12,14}$ Como discutido em vários artigos compilados no número especial da Revista Virtual de Química, dedicada à Química no Antropoceno, ${ }^{22}$ existem diferentes evidências que poderiam marcar o seu início. ${ }^{19-21,23}$

A transição a um novo tempo onde o Sistema Terra se encontra fora do equilíbrio climático típico do Holoceno aconteceu inicialmente de forma gradual a partir do início da agricultura e a domesticação de animais (o chamado "Antropoceno precoce" ou "Early Anthropocene"), passando pela formação de solos antrópicos (Terra Preta de Índio entre outros), também pelo chamado Intercâmbio Colombiano com a chegada dos europeus na América, em 1492, e as suas dramáticas consequências, não só para a vida dos habitantes das Américas como para a globalização e homogeneização das espécies, doenças e rotas comerciais. Mudança que continuou com a Revolução Industrial e, posteriormente, com o crescimento populacional e a mudança exponencial dos parâmetros socioeconômicos, físico-químicos e ambientais a partir da segunda metade do século XX, na chamada Grande Aceleração (Figura 2). ${ }^{19,20,23-25}$

Mesmo existindo muitas evidências de que este último foi o episódio chave, que marcou uma situação quase irreversível de mudanças, ${ }^{26}$ faz-se necessário estabelecer regras para escolher um evento. Assim, a princípio essa escolha deverá ser realizada a partir das assinaturas químicas e físicas deixadas nas rochas e outros estratos geológicos, ou de fósseis, sendo escolhido um marcador (golden spike) que indique claramente o marco inicial e que esteja relacionado a outros indicadores auxiliares sincrônicos em escala global.

\section{A importância da formalização do Antropoceno}

Desde a proposta de Crutzen e Stoermer, o conceito de Antropoceno tem sido crescentemente utilizado, não apenas nas ciências naturais, como também nas ciências sociais, humanas, na política e na economia. ${ }^{2,7,8}$ Do ponto de vista da geologia, o Antropoceno está caracterizado por um conjunto de assinaturas litoestratigráficas, quimioestratigráficas e bioestratigráficas, muitas delas espalhadas na superfície da Terra. Algumas dessas assinaturas podem ser associadas a marcadores estratigráficos já existentes na geologia (como anomalias nas relações isotópicas) e outras são possíveis "novos marcadores", como radionuclídeos artificiais, plásticos, minerais e nanomateriais de origem antrópica. ${ }^{17,27}$ Esses marcadores refletem claramente uma nova fase na história do Sistema Terra que se inicia com a Revolução Industrial e se intensifica marcadamente durante a Grande Aceleração com alterações claras nos ciclos do nitrogênio, fósforo e carbono, impacto sobre a biodiversidade e extinção das espécies e introdução no ambiente continental e marinho de novos materiais, como plásticos, concreto e alumínio, fertilizantes, poluentes derivados de atividades de mineração e radioisótopos derivados do fallout dos testes nucleares atmosféricos. ${ }^{27-31}$ Alguns desses eventos estão limitados a um tempo relativamente curto desde o ponto de vista geológico, mas estão associados a mudanças significativas na história da Terra e que poderão persistir por séculos, milênios ou milhões de anos, ou que serão até mesmo irreversíveis, persistindo após um possível desaparecimento da própria espécie humana.

Desde o ponto de vista das ciências geológicas, o processo de formalização do Antropoceno inclui a necessidade de estabelecer o seu marco inicial. A recente votação do Grupo de Trabalho do Antropoceno, indicando aproximadamente o ano de 1950 e a escolha de um evento de impacto global, como o início dos testes nucleares

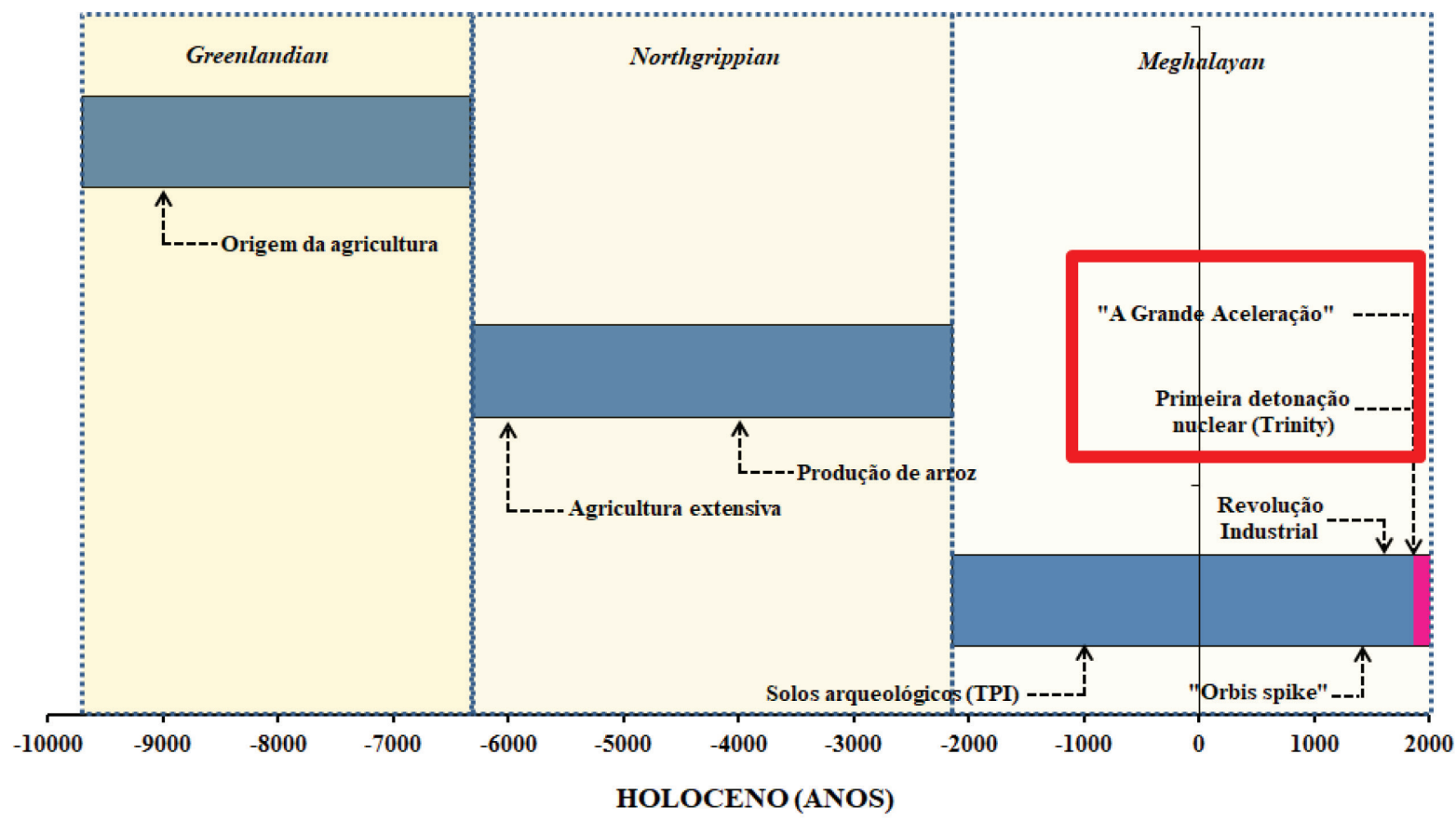

Figura 2. Principais propostas para o início do Antropoceno 
e a Grande Aceleração (episódios aproximadamente coincidentes no tempo) estaria de acordo com a proposta do marco inicial do Holoceno e com a recente (julho de 2018) ratificação da divisão do mesmo em três Idades ou Estágios: Greenlandian (11.700 anos b2k, ou seja, 11.700 anos antes de 2000 d.C.), Northgrippian (8.326 anos b2k, ou seja, 8326 anos antes de 2000 d.C.), e Meghalayan (4200 anos antes de 1950), que correspondem as subséries Holoceno precoce, médio e tardio (Figura 2). ${ }^{20,25,32,33}$

A formalização do Antropoceno promoverá a caracterização dos diferentes estratos na superfície da Terra, especialmente depósitos de origem antrópica, como concreto, plásticos, metais usados na indústria e contaminantes orgânicos como compostos hidrocarbonetos policíclicos aromáticos e, também, terá grande impacto em outras áreas das chamadas ciências do Sistema Terra. ${ }^{23,29-31}$

Essas áreas abrangem, como importantes exemplos, os estudos das mudanças climáticas, acidificação dos oceanos, alterações nos ciclos biogeoquímicos globais, extinção de espécies, diminuição da camada de ozônio, formação de aerossóis, produção e contaminação dos sedimentos e das águas com diferentes poluentes persistentes e outros eventos relacionados aos chamados Limites Planetários. . $3,28,34-37^{-12}$

Mesmo que o termo Antropoceno venha sendo utilizado de maneira informal nessas áreas, o seu reconhecimento e ratificação servirá como base e validação de muitos desses estudos, especialmente os estudos da dinâmica do Sistema Terra.

Finalmente, o reconhecimento formal de que já não é possível esperar que os principais parâmetros relacionados à vida sobre a Terra permaneçam estáveis, poderá ter impacto além das ciências naturais, ${ }^{28}$ em diversos assuntos como planejamento urbano, desenvolvimento, transporte, legislação, políticas de saúde e educação.

\section{POTENCIAIS MARCADORES}

O Grupo de Trabalho do Antropoceno apresentou os marcadores antrópicos potenciais, sendo os escolhidos como mais adequados: novos materiais como plásticos, outros tecnofósseis e partículas relacionadas à queima de combustíveis fósseis - as concentrações de $\mathrm{CO}_{2}$ e a relação isotópica ${ }^{13} \mathrm{C} /{ }^{12} \mathrm{C}$ (ambas relacionadas ao ciclo do carbono) - e marcadores radioativos (fallout relacionado ao primeiro teste nuclear e radionuclídeos artificiais como o isótopo $\left.{ }^{239+240} \mathrm{Pu}\right) .{ }^{11,12}$

\section{Novos materiais antrópicos}

Uma das principais evidências estratigráficas da humanidade são os chamados tecnofósseis, materiais raros ou que não ocorrem na natureza, que são o resultado das atividades humanas e do desenvolvimento tecnológico e permanecem no meio ambiente sem ser degradados substancialmente incorporando-se aos sedimentos, como alumínio, concreto, plásticos e materiais relacionados à industria eletrônica. ${ }^{12,23,29-31}$

Alguns materiais como cerâmica e objetos de cobre acompanharam o desenvolvimento da humanidade durante milênios, mas a sua distribuição no Planeta não é sincrônica e apenas reflete a evolução e as migrações de diferentes povos, como é o caso dos solos arqueológicos amazônicos conhecidos como Terra Preta de Índio que são enriquecidos com fragmentos de cerâmicas, materiais líticos e carvão de origem antrópica. ${ }^{19,20} \mathrm{Em}$ contraste, outros materiais mostram um aumento exponencial na sua produção e acumulação a partir de 1950 , aproximadamente. ${ }^{3,4}$

O alumínio elementar começou ser utilizado no século XIX, mas $98 \%$ de sua produção aconteceu a partir de 1950. O concreto era conhecido e utilizado pelo Império Romano, mas começou a ser usado massivamente a partir da Segunda Guerra Mundial (1939 1945) e aproximadamente metade da sua produção total aconteceu nos últimos 20 - 25 anos. Ambos os materiais, alumínio e concreto estão distribuído pela maior parte da superfície do Planeta. ${ }^{27}$

Hazen e colaboradores catalogaram 208 espécies minerais, aprovadas pela Associação Internacional de Mineralogia, que surgiram como consequência de processos relacionados às atividades humanas. ${ }^{38} \mathrm{~A}$ diversidade e distribuição dos minerais naturais e dos minerais antrópicos são afetadas por diversas atividades humanas, como sua manufatura direta ou através da movimentação de materiais pela mineração, construção de túneis, represas e o transporte de minerais para venda.

A produção mundial de plásticos cresceu, também, rapidamente desde aproximadamente 2 milhões de toneladas em $1950,{ }^{12}$ até 350 milhões de toneladas em 2017. ${ }^{39}$ Calcula-se que de 1950 até 2015 foram fabricadas 7,8 bilhões de toneladas de resinas plásticas, poliéster, poliamidas e acrílicos, sendo que aproximadamente $50 \%$ foi produzido nos últimos 13 anos. ${ }^{40} \mathrm{~A}$ maior parte dos plásticos são usados nas embalagens e a grande maioria não são biodegradáveis de forma que se acumulam no meio ambiente. ${ }^{29,31}$ Estima-se que até 2015 foram geradas 6,3 bilhões de toneladas de resíduos plásticos, $79 \%$ das quais foram acumuladas em lixões, aterros sanitários e no meio ambiente em geral e que provavelmente em 2050, 12 bilhões de toneladas de resíduos plásticos estejam acumulados inadequadamente no ambiente. ${ }^{40}$

Os plásticos têm entrado nos depósitos sedimentares terrestres e aquáticos e eventualmente são incorporados nestes compartimentos, formando plastiglomerados, que resultam da combinação de aglutinados de plásticos, fragmentos de lava, sedimentos marinhos e outros resíduos. ${ }^{41}$ Os plásticos também se acumulam na superfície dos oceanos, especialmente os de baixa densidade, e são transportados pelos ventos e as correntes marinhas podendo chegar inclusive a ilhas remotas. As partículas de microplásticos são ainda mais abundantes e se distribuem mais facilmente que os macroplásticos. As partículas com diâmetros $<1 \mathrm{~mm},{ }^{29,31}$ compostas maioritariamente de microfibras, são muito abundantes e são incorporadas nos sedimentos costeiros em quantidades que oscilam entre dezenas a centenas de fibras por litro de sedimento. ${ }^{42}$ Já os fragmentos mais pesados com densidades $>1 \mathrm{~g} \mathrm{~cm}^{-3}$ afundam, são transportados e finalmente depositam, tendo sido encontrados até em profundidades $>5.000 \mathrm{~m}$. Eventualmente, os microplásticos atingem as regiões polares, tendo sido reportadas quantidades significativas $\left(38-234\right.$ partículas $\left.\mathrm{m}^{-3}\right)$ na camada de gelo do Ártico. ${ }^{43}$

Outras partículas e materiais estão relacionados à queima de combustíveis fósseis. O carbono elementar (black carbon ou BC) é emitido na queima incompleta de combustíveis fósseis e de biomassa vegetal. O BC é inerte, pode ser transportado na atmosfera e acumulado em solos, sedimentos marinhos e lacustres ou na camada de gelo do permafrost, e por ser resistente à degradação pode existir como marcador estratigráfico por dezenas de milhões de anos. Contudo, medições relacionadas em diversos estratos demostraram que os resultados não mostram um padrão sincrônico através do Planeta, refletindo produção e transporte regional, o que não o torna um marcador adequado do início do Antropoceno. ${ }^{12}$

\section{Ciclo do carbono}

As concentrações de $\mathrm{CO}_{2}$ registradas no Observatório Mauna Loa (Havaí, EUA) em maio de 2019, ultrapassaram o valor de 415 ppm (partes por milhão). ${ }^{44}$ Já o último boletim do Programa Global Atmosphere Watch da WMO (World Meteorological Organization) mostrou que em 2018 as concentrações médias de $\mathrm{CO}_{2}$ e $\mathrm{CH}_{4}$ foram $407,8 \pm 0,1$ ppm e $1869 \pm 2$ ppb (partes por bilhão), respetivamente. Esses valores são 147 e $259 \%$ maiores que os da época pré-industrial (antes de 1750). ${ }^{37,45} \mathrm{O}$ ar ocluso no gelo da Groenlândia e da Antártica 
permite determinar quais foram as variações dos gases de efeito estufa (GEE) no passado. Esses registros mostram que, no Pleistoceno, as concentrações foram menores durante os períodos glaciais e maiores durante os períodos interglaciais, com o $\mathrm{CO}_{2}$ variando entre $180 \mathrm{e}$ 280 ppm e o $\mathrm{CH}_{4}$ entre 350 e 700 ppb. $^{14}$

$\mathrm{Na}$ transição do Pleistoceno para o Holoceno os registros no gelo da Antártica mostram um aumento nas concentrações de $\mathrm{CO}_{2}$ de 70 ppm em 6.000 anos (aproximadamente 1 ppm a cada 85 anos). Posteriormente, as concentrações permaneceram aproximadamente estáveis durante todo o Holoceno, com uma pequena diminuição entre 11.000 a 8.000 anos BP e um leve aumento de 260 a 285 ppm a partir de 7.000 BP até a Revolução Industrial. Esse último acréscimo foi atribuído por alguns autores, como Ruddiman, ao desenvolvimento da agricultura (Figura 2), ${ }^{18,46}$ sendo que entre os anos 1570 e 1610 foi observado um decréscimo de 7-10 ppm, atribuído à morte de mais de 50 milhões de habitantes das Américas como consequência da chegada dos conquistadores europeus ao continente, ${ }^{47}$ o que levou a uma significativa diminuição das áreas cultivadas, assim como a perda do conhecimento da formação antropogênica das Terras Pretas de Índio na Amazônia e uma subsequente recuperação, ${ }^{19,20}$ por resiliência, das florestas tropicais (com uma captura estimada em 13.000 milhões de toneladas de $\left.\mathrm{CO}_{2}\right) \cdot{ }^{14,48}$ Contudo, essas mudanças se encontram dentro da variabilidade típica do Holoceno. Por outro lado, a partir de 1850 as concentrações de $\mathrm{CO}_{2}$ começaram aumentar acentuadamente e nos últimos 20 anos essa taxa foi de aproximadamente 2 ppm por ano., ${ }^{1,2}$ Esse aumento é coincidente com os registros de variação isotópica do carbono $\left({ }^{13} \mathrm{C} /{ }^{12} \mathrm{C}\right)$ tanto no $\mathrm{CO}_{2}$ atmosférico como nas plantas e fósseis calcários, atribuído a um acréscimo da proporção de ${ }^{12} \mathrm{C}$ devido ao crescente consumo de combustíveis fósseis. ${ }^{27}$

\section{Fallout nuclear}

Zalasiewicz e colaboradores sugeriram como um possível marcador GSSA para o Antropoceno o primeiro teste nuclear (bomba Trinity A) realizado as 5:30 do dia 16 de julho de 1945 em Alamogordo, Novo México, EUA (Figuras 2 e 3). ${ }^{7}$ Essa escolha, relacionada a um momento histórico, foi considerada equivalente à definição do limite Cretáceo-Paleogeno que mesmo tendo sido definido por GSSP, historicamente é considerado tão relevante como o momento do impacto do meteorito na península de Yucatan. ${ }^{49}$ Todavia, essa assinatura é geograficamente limitada e não poderia ser um marcador GSSP sincronicamente adequado.

Em contraste, como será discutido nos próximos itens, o fallout relacionado aos testes nucleares atmosféricos iniciados em 1952 promoveu a deposição de grande quantidade de radionuclídeos no meio ambiente, o que pode fornecer um marcador sincrônico que difere em apenas sete anos do Teste Trinity (Figura 3). ${ }^{1,12,50,51}$

\section{FONTES DE RADIAÇÃO ANTRÓPICA}

Atualmente, existem inúmeras fontes de radiação naturais e antrópicas não relacionadas às armas atômicas ou aos testes nucleares. As maiores contribuições à radiação natural são os raios cósmicos de alta energia incidentes na atmosfera terrestre e os radionuclídeos originados no interior da crosta terrestre. ${ }^{50}$ As atividades humanas modificam a exposição à radiação natural, especialmente através das atividades de mineração, produção de fertilizantes, uso e combustão do petróleo e seus derivados. Todos os organismos vivos estão expostos a essa radiação, parte dela em forma aproximadamente constante
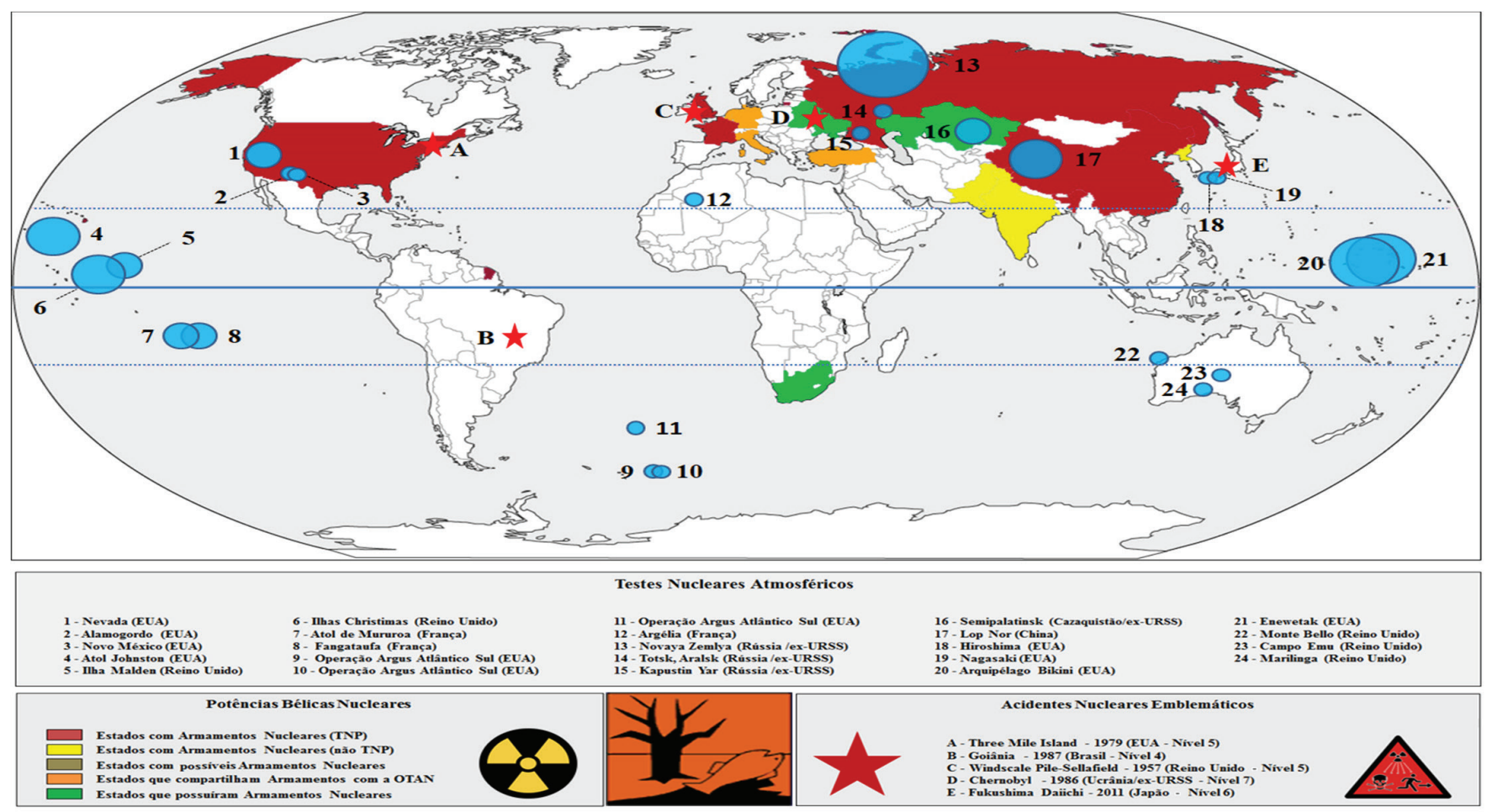

RENDIMENTO DAS EXPLOSÓES DOS TESTES NUCLEARES ATMOSFÉRICOS GLOBAIS (MEGATONELADAS DE TRINITROTOLUENO)
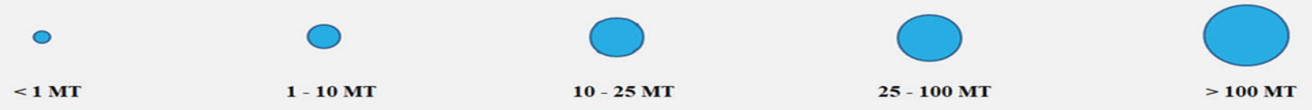

Figura 3. Panorama global de significativos acidentes nucleares, localização de testes nucleares atmosféricos e intensidade das detonações. Adaptado e modificado da literatura $1,12,50,51$ 
e uniforme, como por exemplo, a ingestão do potássio (isótopo ${ }^{40} \mathrm{~K}$ ) pelos alimentos. Outras formas de exposição à radiação variam dependendo da localização geográfica e das atividades ocupacionais, sendo maior, por exemplo, para trabalhadores de minas subterrâneas ou processos industriais mínero-metalúrgicos. A exposição à radiação cósmica, por exemplo, é mais acentuada em maiores altitudes e as concentrações de urânio e tório são diferentes para diversas regiões do Planeta. ${ }^{50,51}$

A primeira fonte antrópica registrada de radiação foi para o uso medicinal a partir do final do século XIX e é considerada atualmente a maior fonte de exposição artificial, porém restrita a determinados indivíduos (com fins de diagnósticos ou de tratamento) e que, em geral, não atinge o meio ambiente de forma que não são obtidos registros geológicos, exceto pelo descarte inadequado de resíduos nucleares de serviços de saúde ou extravio de equipamentos em condições não controladas, ${ }^{1,50}$ como ocorrido no incidente com o ${ }^{137}$ Césio em Goiânia (Figura 3). A radiação recebida é medida em unidades de $\mathrm{Sv}$ (sievert). Tipicamente, a exposição anual total à radiação natural é de 1-10 mSv (milisievert), enquanto que o valor médio de exposição anual para exames médicos é de $0,04-1,0 \mathrm{mSv} .{ }^{50}$

Além dessas fontes, existe a contribuição da energia nuclear. A primeira planta industrial comercial de energia nuclear foi instalada em 1956 em Calder Hall, Inglaterra. ${ }^{52} \mathrm{O}$ uso da energia nuclear aumentou rapidamente entre 1970 e 1985, até o acidente de Chernobyl no dia 26 de abril de 1986 (Figura 3). Esse é considerado o acidente mais grave acontecido na história da indústria da energia nuclear e nele foram liberados, principalmente, os isótopos ${ }^{131} \mathrm{I},{ }^{134} \mathrm{Cs}$ e ${ }^{137} \mathrm{Cs}$. O tempo de vida-média do isótopo ${ }^{131} \mathrm{I}$ é de apenas oito dias, mas contaminou vegetais e outros alimentos posteriormente ingeridos pela população ucraniana e de outras repúblicas da então União das Repúblicas Socialistas Soviéticas (URSS). Já os isótopos de césio têm maiores tempos de vida-média (2 e 30 anos, para o ${ }^{134} \mathrm{Cs}$ e ${ }^{137} \mathrm{Cs}$, respectivamente). Os gases e partículas radiativas foram transportados à grandes distâncias pelo vento e depositados pela chuva, afetando outros países do continente Europeu. ${ }^{50,52} \mathrm{O}$ acidente de Fukushima Daiichi, em março de 2011, quando a usina foi atingida por um tsunami, é considerado o segundo mais impactante acidente nuclear após Chernobyl, e nele também foram liberados ${ }^{131} \mathrm{I}$ e ${ }^{137} \mathrm{Cs}$ (Figura 3). O material radiativo foi emitido na atmosfera e transferido ao solo e às águas do Oceano Pacífico por deposição seca e úmida. Além disso, a água contaminada foi despejada diretamente no litoral, contaminando as águas do oceano Pacífico e parte da costa do Japão. ${ }^{53}$

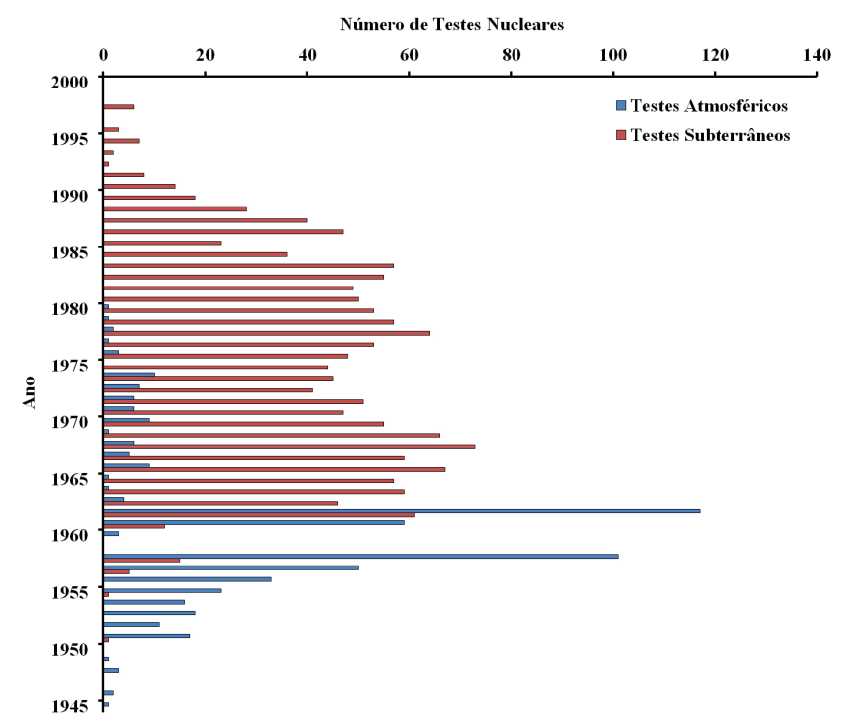

O uso da energia nuclear envolve a mineração e tratamento de urânio para a produção do combustível nuclear para os reatores, o armazenamento e tratamento dos resíduos radiativos, a reciclagem dos materiais e descarte dos resíduos, além do transporte dos materiais radiativos. ${ }^{50,52}$ Geralmente, a contaminação causada por esses processos está localizada no hemisfério norte, na costa do Oceano Atlântico, e na costa do Japão (Mar do Japão).

A desintegração do satélite SNAP-9A em 1964 causou a dispersão de plutônio (isótopo ${ }^{238} \mathrm{Pu}$ ), principalmente no hemisfério Sul, e poderia ser uma fonte importante para datação, mas por um tempo muito limitado, já que a vida média deste isótopo é de apenas 88 anos. ${ }^{1}$

Todos esses eventos envolvendo acidentes nucleares são candidatos muito limitados para serem usados como golden spikes do Antropoceno porque seus impactos são considerados de alcance regional. Em contraste e, como será discutido no próximo item, os testes de armas nucleares, no período de 1945 a 1980, e seu uso efetivo em Hiroshima e Nagasaki em agosto de 1945, forneceram marcadores em nível global em solos e sedimentos. ${ }^{1}$

\section{Testes com armas nucleares}

Como demonstrado na Figura 4, foram realizados 2.053 testes com armas nucleares no período entre 1945 e 1980, principalmente na Ásia Central, oceano Pacífico e Oeste dos Estados Unidos, dos quais 543 foram realizados na atmosfera. ${ }^{1,50} \mathrm{~A}$ maior parte dos testes foram realizados nos períodos entre 1951 e 1958 e reiniciados entre 1961 e 1962 , após a moratória de $1959 .{ }^{50,52}$ Os maiores testes na atmosfera (> 4 MT), foram realizados pela China (Lop Nor, 1976), Estados Unidos (Bikini, Enewetak, Johnston e Christmas Islands entre 1952 e 1962), e União Soviética (Novaya Zemlya entre 1961 e 1962), totalizando 25 testes que respondem por aproximadamente $66 \%$ do poder total explosivo e $55 \%$ da emissão radiativa (Figura 3). Por outro lado, os testes subterrâneos aconteceram entre 1962 e $1990 .^{50,52}$

Na Figura 1S (Material Suplementar) são mostrados em forma resumida os países que realizaram testes nucleares. Como ilustrado nas Figuras 3 e 1S, os testes nucleares foram conduzidos por apenas cinco países, mas em diversos locais do planeta, tanto na atmosfera quanto em áreas subterrâneas, incluindo torres, superfície do oceano, balões, aviões e lançamentos desde plataformas em terrenos elevados. Além disso, como pode ser observado nas Figuras 4 e 1S, deve-se destacar que os testes nucleares aconteceram principalmente no momento da chamada "Guerra Fria", na qual as principais potências detentoras

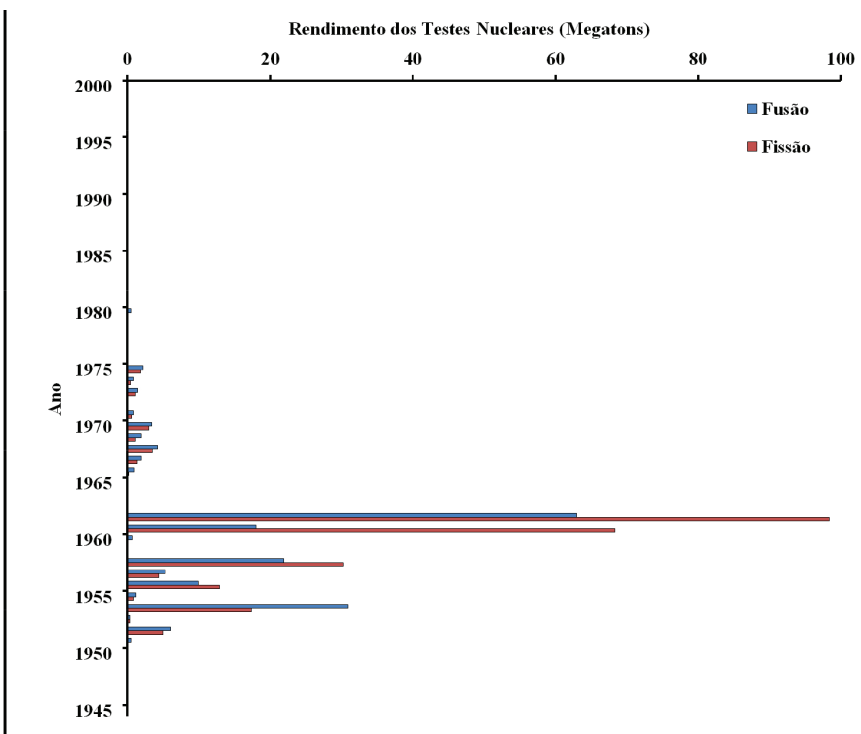

Figura 4. Distribuição dos testes nucleares atmosféricos e subterrâneos e rendimentos dos testes nucleares de fusão e fissão 
de artefatos bélicos nucleares disputavam a hegemonia geo-política no mundo e que estes mesmos testes serviam como demonstração de força e efeito de propaganda do desenvolvimento tecnológico, tanto do regime capitalista (Estados Unidos da América, Reino Unido e França) quanto do regime socialista (União das Repúblicas Socialistas Soviéticas e China) (Figura 1S). ${ }^{52}$

Dependendo da latitude, longitude e dos fatores meteorológicos, o fallout nuclear foi distribuído em escala local, regional ou global. ${ }^{52}$ No caso dos testes na superfície, parte do material nuclear foi depositado nas áreas próximas (fallout local) e outra parte foi transportada pelos ventos a milhares de quilómetros (fallout intermediário), dependendo dos fatores meteorológicos, altitude e local em que foi realizado. ${ }^{50}$ Para alguns elementos, como os isótopos ${ }^{95} \mathrm{Zr}$ e ${ }^{144} \mathrm{Ce}$, aproximadamente $50 \%$ do fallout foi depositado localmente e $25 \%$ em nível regional. Para outros radionuclídeos como ${ }^{90} \mathrm{Sr},{ }^{137} \mathrm{Cs}$ e ${ }^{131} \mathrm{I}$, cerca de $50 \%$ em média foi depositado regionalmente e o restante disperso na atmosfera cobrindo grandes distâncias.

A distribuição geográfica dos radionuclídeos associados ao fallout nuclear, devido aos testes, foi medida principalmente a partir dos isótopos ${ }^{90} \mathrm{Sr} \mathrm{e}{ }^{137} \mathrm{Cs} .{ }^{1,52} \mathrm{O}$ fallout do ${ }^{90} \mathrm{Sr}$ está concentrado nas latitudes intermediárias $\left(30^{\circ}-60^{\circ}\right)$ em cada hemisfério e é consideravelmente menor nas regiões polares e na linha do equador. Livinsgton e Povinec, estimaram a partir de determinações de ${ }^{90} \mathrm{Sr},{ }^{137} \mathrm{Cs}$ e ${ }^{239} \mathrm{Pu}$ que, no ambiente marinho, aproximadamente $76 \%$ das fontes de radiação estão localizadas no hemisfério norte, e são provenientes do fallout global ocasionados pelos testes e acidentes nucleares, inclusive de aviões e embarcações de despejo de resíduos radiativos. ${ }^{54}$

\section{OS POSSÍVEIS MARCADORES DO ANTROPOCENO}

Para serem marcadores adequados para definir o início do Antropoceno, os isótopos radiativos devem reunir uma série de condições: serem ausentes ou escassos no ambiente natural (ou seja, ser de origem antrópica), ter tempos de vida-média longos para fornecer um sinal suficientemente duradouro, ter baixa mobilidade e solubilidade e alta radioatividade para estarem fixados nos sedimentos e se encontrarem em abundancias suficientes para serem facilmente detectáveis e mensurados. ${ }^{1}$

Alguns dos isótopos mais abundantes têm tempos de meia-vida muito curtos, como o ${ }^{137} \mathrm{Cs}$ (30 anos), o ${ }^{3} \mathrm{H}$ (trítio) (12 anos), o ${ }^{90} \mathrm{Sr}$ (29 anos) e o ${ }^{241} \mathrm{Pu}$ (14 anos) o que limita sua potencial utilidade como marcadores para tempos maiores que um século. ${ }^{1,12,21}$ Outros radioisótopos têm maiores tempos de vida-média, como o ${ }^{241} \mathrm{Am}$ (432 anos), o ${ }^{14} \mathrm{C}$ (5.700 anos) e o ${ }^{239} \mathrm{Pu}\left(24.110\right.$ anos). ${ }^{1,12}$

$\mathrm{O}{ }^{14} \mathrm{C}$ tem um tempo de meia-vida razoavelmente longo e é um dos radionuclídeos mais abundantes, mostrando um pico nos anos 1963 e 1964 na maioria dos reservatórios, incluindo solos, troncos de árvores e corais, com um sinal suficientemente claro para ser utilizado como potencial GSSP, tal como sugerido por Lewis e Maslin. ${ }^{24}$ No Material Suplementar (Figura 2S) é mostrada a variação da concentração atmosférica do ${ }^{14} \mathrm{C}$ durante o Holoceno. ${ }^{55}$

Sobre essa indicação, é importante notar que contrariamente ao habitual (colocar como GSSP o declínio de um sinal), seria colocado o máximo. Um dos principais problemas do isótopo ${ }^{14} \mathrm{C}$ é sua alta mobilidade em muitos sedimentos e, também, ser um sinal diacrônico, localizado principalmente no hemisfério norte e atenuado e deslocado no tempo no hemisfério sul. Estudos da relação isotópica ${ }^{14} \mathrm{C} /{ }^{12} \mathrm{C}$ em corais no hemisfério norte (Florida e Bermudas), perto do local onde foram realizados alguns testes atômicos mostram que o aumento no sinal do ${ }^{14} \mathrm{C}$ começa aproximadamente em 1955, com um máximo entre 1963 e 1964, enquanto que no hemisfério sul (arquipélago de Abrolhos, Brasil) começa no final da década de 1950 com um máximo em 1974. ${ }^{56}$
Os isótopos do plutônio têm baixa solubilidade e alta radiatividade, o que facilita sua associação à argila e à matéria orgânica. Além disso, o tempo de vida-média do ${ }^{239} \mathrm{Pu}$ permite que este seja utilizado por mais de 100.000 anos, considerando o atual desenvolvimento dos métodos espectrográficos. Já o ${ }^{240} \mathrm{Pu}$, menos abundante e com um tempo de vida-média de 6.563 anos poderia ser detetado por um tempo menor.

As concentrações de plutônio (a soma dos dois isótopos) mostram um máximo entre 1963 e 1964 e a partir dessa data um claro declínio como consequência dos tratados de banimento de armas nucleares. Atualmente, a única fonte são as emissões atmosféricas controladas das plantas industriais nucleares, fábricas de reprocessamento de combustível nuclear, desintegração atmosférica de geradores elétricos de satélites, emissões de fábricas voltadas para a manutenção e produção de componentes de armas atômicas, ressuspensão de partículas do solo e eventuais acidentes nucleares. ${ }^{52,57,58} \mathrm{Em}$ ambientes terrestres, o plutônio se associa com frações geoquímicas específicas como óxidos de ferro e manganês e ácidos húmicos e, portanto tende a ser relativamente imóvel em sedimentos (inclusive gelo). ${ }^{58}$

Nos oceanos se associa ao material em suspensão e movimenta-se nas colunas d'água, de forma que sua distribuição é afetada pelas correntes marinhas e pelo movimento dos sedimentos. Muitos golden spikes se encontram em sedimentos de áreas costeiras porque tendem a ser mais contínuos que os estratos terrestres e contêm registros da vida de plantas e animais que podem ser relacionados em várias localidades. Em oceano aberto, parte do plutônio se encontra ainda nas águas e desce lentamente até os sedimentos. No caso do amerício o depósito é mais rápido, mas esse radioisótopo se encontra em concentrações menores e tem um tempo de vida-média curto, pelo qual não tem sido considerado um bom marcador. ${ }^{12}$

Em uma revisão publicada em 2012,59 Sanders e colaboradores analisaram as atividades detectadas para ${ }^{239+240} \mathrm{Pu}$ e ${ }^{137} \mathrm{Cs}$ em sedimentos de áreas costeiras do Brasil. As atividades para plutônio foram determinadas em um manguezal de Paraty (Rio de Janeiro) ${ }^{58}$ e em sedimentos superficiais das costas das regiões norte, sul, nordeste e sudeste. ${ }^{59-62} \mathrm{Já}$ as atividades máximas para ${ }^{137} \mathrm{Cs}$ foram determinadas por espectrometria de radiação gama em testemunhos de sedimentos costeiros brasileiros em várias regiões. ${ }^{60,61,63-68} \mathrm{Já}$ em outro artigo recente publicado em 2016, Ferreira e colaboradores avaliaram a ocorrência e distribuição de ${ }^{137} \mathrm{Cs}$ nos sedimentos costeiros do Atlântico Sul $\left(0^{\circ}-35^{\circ} \mathrm{S}\right)$ e na Baia do Almirantado (Antartica). ${ }^{69,70}$ Os resultados obtidos mostram que o fallout do ${ }^{137} \mathrm{Cs}$ está diretamente relacionado aos testes nucleares com um máximo em 1963 em excelente concordância com os resultados obtidos no hemisfério norte. Ferreira e colaboradores concluíram nesse trabalho que ${ }^{137} \mathrm{Cs}$ seria um excelente marcador a prazos curtos e médios (os autores sugerem até 7 - 10 meias-vidas, ou seja até o século XXIII) nas atuais condições de análise instrumental.

No hemisfério norte existem registros para ${ }^{239+240} \mathrm{Pu}$ em numerosos ambientes que confirmam o pico em 1963, como sedimentos marinhos e lacustres, corais, registros de gelo no Ártico e glaciares. ${ }^{71-73} \mathrm{As}$ regiões lacustres, onde os depósitos têm pouca mobilidade, e polares, onde a acumulação é rápida e pouco sujeita à intervenção, mostram-se como uma excelente alternativa para a localização do golden spike. No caso da datação do Holoceno e suas divisões em idades, como já foi mencionado, foi utilizada essa alternativa através de testemunhos de gelo da Groenlândia. ${ }^{16,19,20}$

Existem evidências de que o plutônio é imobilizado nos registros de gelo, tendo sido possível fazer determinações de alta resolução em gelos polares e em latitudes médias, por exemplo, em glaciares alpinos da Itália e da Suíça. Essas medições em glaciares mostram os três períodos principais nos testes nucleares: o aumento da radiação a partir de 1954 - 1955, até um primeiro máximo em 1958, um 
decréscimo nos dois anos seguintes e um novo acréscimo a partir de 1961 - 1962 com um máximo claro em 1963 (40\% mais intenso que o pico de 1958). Após a assinatura do tratado parcial entre os Estado Unidos da América e a União das Repúblicas Socialistas Soviéticas em $1964,{ }^{52}$ a deposição de plutônio teve um novo decréscimo atingindo um novo mínimo em 1967. No período entre 1967 e 1975 foram observados alguns novos picos máximos (aproximadamente 20 a $30 \%$ do valor de 1964) que foram atribuídos ao transporte de areias contaminadas do deserto do Sahara como consequência dos testes realizados pela França na década de 1960 na Argélia (Figura 3). ${ }^{73}$ Esses resultados estão de acordo com os obtidos em outras regiões, por exemplo no glaciar Belukha (Siberia) ${ }^{74}$ Apesar dos registros de gelo terem se mostrado uma ótima opção como golden spikes, existe a preocupação que o derretimento de glaciares e calotas polares, devido ao aquecimento global, leve à perda desses registros. ${ }^{1,19,20}$

\section{CONCLUSÕES}

O conceito de Antropoceno está relacionado às evidências que apontam que as atividades humanas mudaram substancialmente o Sistema Terra, de maneira que as mudanças observadas encontram-se fora da variabilidade típica do Holoceno e, além disso, a velocidade dessas mudanças leva a consequências globais ainda desconhecidas. Já a definição do Antropoceno com uma nova Época no contexto da Geologia envolve critérios geocronológicos e cronoestratigráficos. Desde o ponto de vista geocronológico às mudanças nos parâmetros sócio-econômicos e físico-químicos a partir do ano 1950, que caracterizariam a chamada Grande Aceleração, seriam provavelmente o mais importante indicador. Contudo, esses eventos, principalmente o aumento das concentrações de $\mathrm{CO}_{2}$ e o aparecimento de novos materiais, são considerados indicadores pobres para ser claramente um marcador GSSP. Por outro lado, o desenvolvimento da energia nuclear não é por si só um indicador suficiente do ponto de vista geocronológico, mas ao registro sedimentar do fallout atmosférico coincidir com outros eventos, fornece um excelente indicador cronoestratigráfico.

Em particular, o aparecimento da assinatura do ${ }^{239+240} \mathrm{Pu}$ no início da década de 1950 e um máximo claro em 1963, fornecem um excelente GSSP. Outros isótopos radiativos como ${ }^{137} \mathrm{Cs}$, ${ }^{14} \mathrm{Ce} \mathrm{e}^{241} \mathrm{Am}$ podem ser usados como assinaturas ou marcadores antrópicos auxiliares.

Após o voto vinculante do Grupo de Trabalho do Antropoceno, em maio de 2019, novas discussões e pesquisas deverão ser realizadas para coletar as evidencias suficientes que deverão ser apresentadas para a votação final da Subcomissão de Estratigrafia do Quaternário, da Comissão Internacional de Estratigrafia e ratificação da União Internacional de Ciências Geológicas.

\section{MATERIAL SUPLEMENTAR}

As Figuras 1S e 2S estão disponíveis no website da revista Química Nova (http://quimicanova.sbq.org.br) com acesso livre.

\section{AGRADECIMENTOS}

Os autores agradecem o apoio financeiro de CNPq e FAPERJ.

\section{REFERÊNCIAS}

1. Waters, C. N.; Syvitski, J. P. M.; Galuszka, A.; Hancock, G. J.; Zalasiewicz, J.; Cearreta, A.; Grinevald, J.; Jeandel, C.; McNeill, J. R.; Summerhayes, C.; Barnosky, A.; Bull. At. Sci. 2015, 71, 46.

2. Veiga, J. E.; O Antropoceno e a Ciência do Sistema Terra, Editora 34: São Paulo, 2019.
3. Ellis, E. C.; Anthropocene: A Very Short Introduction, UOP Oxford: Oxford, 2018.

4. McNeill, J. R.; The Great Acceleration: An Environmental History of the Anthropocene since 1945, Harvard University Press: Cambridge, 2016.

5. Schwägerl, C.; Jones, L. R.; The Anthropocene: The Human Era and How It Shapes Our Planet, Synergetic Press: Londres, 2014.

6. Davies, J.; The Birth of the Anthropocene, University of California Press: California, 2016.

7. Crutzen, P. J.; Stoermer, E. F.; Global Change Newsletter 2000, 41, 17.

8. Silva, C. M.; Arbilla, G.; Rev. Virtual Quim. 2018, 10, 1619.

9. http://quaternary.stratigraphy.org/working-groups/anthropocene/, acessada em Março 2020.

10. http://www.35igc.org/, acessada em Março 2020.

11. Zalasiewicz, J.; Waters, C. N.; Summerhayes, C. P.; Wolfe, A. P.; Barnosky, A. D.; Cearreta, A.; Crutzen, P.; Ellis, E.; Fairchild, I. J.; Galuszka, A.; Haff, P.; Hajdas, I.; Head, M. J.; Ivar do Sul, J. A.; Jeandel, C.; Leinfelder, R.; McNeill, J. R.; Neal, C.; Odada, E.; Oreskes, N.; Steffen, W.; Syvitski, J.; Vidas, D.; Wagreich, M.; Williams, M.; Anthropocene 2017, 19, 55.

12. Zalasiewicz, J.; Waters, C.; Williams, M.; Summerhayes, C.; The Anthropocene as a Geological Time Unit: A Guide to the Scientific Evidence and Current Debate, Cambridge University Press: Cambridge, 2019.

13. http://quaternary.stratigraphy.org/wp-content/uploads/2018/12/ Anthropocene-Working-Group-Newsletter-Vol-8.pdf, acessada em Março 2020.

14. Lewis, S. L.; Maslin, M. A.; The Human Planet: How We Created the Anthropocene, Yale University Press: Londres, 2018.

15. http://www.stratigraphy.org/index.php/ics-chart-timescale, acessada em Março 2020.

16. Walker, M.; Johnsen, S.; Rasmussen, S. O.; Popp, T.; Steffensen, J.-P.; Gibbard, P.; Hoek, W.; Lowe, J.; Andrew, J.; Bjorck, S.; Cwynar, L. C.; Hughen, K.; Kershaw, P.; Kromer, B.; Litt, T.; Lowe, D. J.; Nakagawa, T.; Newnham, R.; Schwander, J.; Journal of Quaternary Science 2009, 24, 3.

17. Zalasiewicz, J.; Waters, C. N.; Williams, M.; Barnosky, A. D.; Cearreta, A.; Crutzen, P.; Ellis, E.; Ellis, M. A.; Fairchild, I. J.; Grinevald, J.; Haff, P. K.; Hajdas, I.; Leinfelder, R.; McNeill, J.; Odada, E. O.; Poirier, C.; Ritcher, D.; Steffen, W.; Summerhayes, C.; Syvitski, J. P. M.; Vidas, D.; Wagreich, M.; Wing, S. L.; Wolfe, A. P.; Zhisheng, A.; Oreskes, N.; Quaternary International 2015, 383, 196.

18. Ruddiman, W. F.; PPG: Earth and Environment 2018, 42, 451.

19. Soares, R.; Maddock, J. E. L.; Campos, D. V. B.; Madari, B. E.; Machado, P. L. O. A.; C Santelli, R. E.; Rev. Virtual Quim. 2018, 10, 1659 .

20. Soares, R.; Maddock, J. E. L.; Campos, D. V. B.; Madari, B. E.; Machado, P. L. O. A.; Santelli, R. E.; Rev. Virtual Quim. 2018, 10, 1693.

21. Godoy, J. M.; Rev. Virtual Quim. 2018, 10, 1733.

22. Silva, C. M.; Arbilla, G.; Machado, W.; Soares, R.; Rev. Virtual Quim. 2018, 10, 1618.

23. Martins, J. R. S.; Montagner, C. C.; Rev. Virtual Quim. 2018, 10, 1719.

24. Lewis, S. L.; Maslin, M. A.; Nature 2015, 519, 171.

25. Silva, C. M.; Arbilla, G.; Soares, R.; Machado, W.; Rev. Virtual Quim. 2018, 10, 1648.

26. Steffen, W.; Broadgate, W.; Deutsch, L.; Gaffney, O.; Ludwig, C.; Anthropocene Rev. 2015, 2, 81.

27. Waters, C. N.; Zalasiewicz, J.; Summerhayes, C.; Barnosky, A. D.; Poirier, C.; Gałuszka, A.; Cearreta, A.; Edgeworth, M.; Ellis, E. C.; Ellis, M.; Jeandel, C.; Leinfelder, R.; McNeill, J. R.; Richter, D.; Steffen, W.; Syvitski, J.; Vidas, D.; Wagreich, M.; Williams, M.; Zhisheng, A.; Grinevald, J.; Odada, E.; Oreskes, N.;Wolfe, A. P.; Science 2016, 6269, 2622.

28. Miranda, J. L.; Gomes, F.; de Almeida, C. D.; Gerpe, R.; Rev. Virtual Quim. 2018, 10, 1990. 
29. G Olivatto, G. P.; Carreira, R.; Tornisielo, V. L.; Montagner, C. C.; Rev. Virtual Quim. 2018, 10, 1968.

30. Afonso, J. C.; Rev. Virtual Quim. 2018, 10, 1849.

31. Hatje, V.; da Cunha, L. C.; Costa, M. F.; Rev. Virtual Quim. 2018, 10, 1947.

32. http://www.stratigraphy.org/index.php/ics-news-and-meetings/120-icschart-containing-the-quaternary-gssps-and-new-stages-v-2018-07-isnow-released, acessada em Março 2020.

33. Walker, M. J. C.; Berkelhammer, M.; Björck, S.; Cwynar, L. C.; Fisher, D. A.; Long, A. J.; Lowe, J. J.; Newnham, R. M.; Rasmussen, S. O.; Weiss H.; Journal of Quaternary Science 2012, 27, 649.

34. Rockström, J.; Steffen, W.; Noone, K.; Persson, Å., Chapin, III, F. S.; Lambin, E.; Lenton, T. M.; Scheffer, M.; Folke, C.; Schellnhuber, H.; Nykvist, B.; De Wit, C. A.; Hughes, T.; van der Leeuw, S.; Rodhe, H.; Sörlin, S.; Snyder, P. K.; Costanza, R.; Svedin, U.; Falkenmark, M.; Karlberg, L.; Corell, R. W.; Fabry, V. J.; Hansen, J.; Walker, B.; Liverman, D.; Richardson, K.; Crutzen, P.; Foley, J.; Ecol. Soc. 2009, 14,32 .

35. Rockström, J.; Steffen, W.; Noone, K.; Persson, A.; Chapin III, F. S.; Lambin, E. F.; Lenton, T. M.; Scheffer, M.; Folke, C.; Schellnhuber, H. J.; Nykvist, B.; de Wit, C. A.; Hughes, T.; van der Leeuw, S.; Rodhe, H.; Sörlin, S.; Snyder, P. K.; Costanza, R.; Svedin, U.; Falkenmark, M.; Karlberg, L.; Corell, R. W.; Fabry, V. J.; Hansen, J.; Walker, B.; Liverman, D.; Richardson, K.; Crutzen, P.; Foley, J. A.; Nature 2009, $461,472$.

36. Steffen, W.; Richardson, K.; Rockstrom, J.; Cornell, S. E.; Fetzer, I.; Bennett, E. M.; Biggs, R.; Carpenter, S. R.; de Vries, W.; de Wit, C. A.; Folke, C.; Gerten, D.; Heinke, J.; Mace, G. M.; Persson, L. M.; Ramanathan, V.; Reyers, B.; Sorlin, S.; Science 2015, 347, 1259855-1.

37. Miranda, J. L.; Moura, L. C; de Ferreira, de H. B. P.; Abreu, T. P.; Rev. Virtual Quim. 2018, 10, 1915.

38. Hazen, R. M.; Grew, E. S.; Origlieri, M. J.; Downs, R. T.; Am. Mineral. 2017, 102, 595

39. https://www.plasticseurope.org/en/resources/publications/619-plasticsfacts-2018,acessada em Março 2020.

40. Geyer, R.; Jambeck, J. R.; Law, K. L.; Sci. Adv. 2017, 3 , 1.

41. Corcoran, P. L.; Moore, C. J.; Jazvac, K.; GSA Today 2014, $24,4$.

42. Obbard, R. W.; Sadri, S.; Wong, Y. Q.; Khitun, A. A.; Baker. I.; Thompson, R. C.; Earth's Future 2014, 2, 315.

43. Browne, M. A.; Crump, P.; Niven, S. J.; Teuten, E.; Tonkin, A.; Galloway, T.; Thompson, R.; Environ. Sci. Technol. 2011, 45, 9175.

44. https://www.co2.earth/daily-co2, acessada em Março 2020.

45. https://library.wmo.int/index.php?lvl=notice_display\&id=21620, acessada em Março 2020.

46. Ruddiman, W. F.; Clim. Change 2003, 61, 261.

47. Koch, A.; Brierley, C.; Maslin, M. M.; Lewis, S. L.; Quat. Sci. Rev. 2019, 207, 13.

48. Bauska, T. K.; Joos, F.; Mix, A. C.; Ahn, J.; Brook, E. J.; Nat. Geosci. 2015, 8, 383.

49. Molina, E.; Alegret, L.; Aremillas, I.; Arz, J.A.; Gallala, N.; Hardenbol, J.; von Salis, K.; Steuerbaut, E.; Vandenberghe, N.; Zagbib-Turki, D.; Episodes 2006, 29, 263.

50. https://www.unscear.org/unscear/en/publications/2000_1.html, acessada em Março 2020.
51. https://www.unscear.org/docs/publications/1982/UNSCEAR_1982_ Report.pdf, acessada em Março 2020.

52. Marques, A.; Energia nuclear e adjacências. EdUERJ: Rio de Janeiro, 2010.

53. Povinec, P. P.; Hirose, K.; Aoyama, M.; Radionuclide releases into the environment. Fukushima Accident: Radioactivity Impact on the environment, Elsevier: Londres 2013.

54. Livinsgton, H. D.; Povinec, P. P.; Ocean \& Coastal Management 2000 , 8,689 .

55. https://www.unscear.org/docs/publications/2016/UNSCEAR_WP_2016. pdf., acessada em Março 2020.

56. Druffel, E. R.; Radiocarbon 1996, 38, 563

57. Hancock, G. J.; Tims, S. G.; Fifield, L. K.;Webster, I. T.; Geol. Soc. 2014, 395, 28.

58. Sanders, C. J.; Smoak, J. M.; Sanders, L. M.; Waters, M. N.; Patchineelam, S. R.; Ketterer, M. E.; J. Radioanal. Nucl. Chem. 2010, 283, 593.

59. Sanders, C.J.; Patchineelam, S. R.; Machado, W.; Silva-Filho, E. V.; Albuquerque, A. L. S.; Caldeira, P. P.; Sanders, L. M.; Quim. Nova 2012, 36, 1209.

60. Cunha, I.; Figueira, R. C. L.; Saito, R. T.; J. Radioanal. Nucl. Chem. 1999, 239, 3477.

61. Figueira, R. C. L.; Tessler, M. G.; de Mahiques, M. M.; Cunha, I. I. L.; Sci. Total Environ. 2006, 357, 146.

62. Figueira, R. C. L.; Furtado, V. V.; Tessler M. G.; Cunha, I. I. L.; Brazilian Journal of Oceanography 2003, 51, 55.

63. Gomes, C. F.; Godoy, J. M.; Godoy, M. L. D. P.; Carvalho, Z. L.; Lopes, R. T.; Sanchez-Cabeza, J.; Osvath, I.; Lacerda, L. D.; J. Environ. Radioact. 2011, 102, 871.

64. Sanders, C. J.; Smoak, J. M.; Sathy, M.; Araripe, D. E.; Sanders, L. M.; Patchineelam, S.; R. Environ. Earth Sci. 2010, 60, 1291.

65. Martins, C. C.; Bícego, M. C.; Mahiques, M. M.; Figueira, R. C. L.; Tessler, M. G.; Montone, R. C.; Environ. Pollut. 2010, 158, 3355.

66. Sanders, C. J.; Santos, I. R.; Silva, E. V.; Patchineelam, S. R.; Mar. Pollut. Bull. 2006, 52, 1089.

67. Godoy, J. M.; Carvalho, Z. L.; Fernandes, F. C.; Danelon, O. M.; Ferreira, A. C. M.; Roldão, L. A.; J. Environ. Radioact. 2003, 70, 193.

68. Saito, R. T.; Figueira, R. C. L.; Tesslier, M. G.; Cunha, I. I. L.; J. Radioanal. Nucl. Chem. 2001, 250, 109.

69. Ferreira, P. A. de L., Figueira, R. C. L., Siegle, E., Neto, N. E. A., Martins, C. de, C., Schettini, C. A. F.; Anthropocene 2016, 14, 34

70. Ferreira, P. A. L.; Ribeiro, A. P.; Nascimento, M. G.; Martins, C. C.; Mahiques, M. M.; Montone, R. C.; Figueira, R. C. L.; Sci. Tot. Environ. 2013, 443, 505

71. Lindahl, P.; Asami, R.; Iryu, Y.; Worsfold, P.; Roach, M. K.; Choi, M. S.; Geochim. Cosmochim. Acta 2011, 75, 1346.

72. Wendel, C. C.; Oughton, D. H; Lind, O. C; Skipperud, L; Fifield, L. K; Isaksson, E.; Tims, S. G.; Salbu, B.; Sci. Total Environ. 2013, 461, 734.

73. Gabrieli, J.; Cozzi, G.; Vallelonga, P.; Schwikowski, M.; Sigl, M.; Eickenberg, J.; Wacker, L.; Boutron, C.; Gaeggeler, H.; Cescon, P.; Barbante, C.; Atmos. Environ. 2011, 45, 587.

74. Olivier, S.; Bajo, S.; Fifield, L. K.; Gaggeler, H. W.; Papina, T.; Santschi, P. H.; Schotterer, U.; Wacker, L.; Environ. Sci. Technol. 2005, 38, 6507. 